EPJ manuscript No.

(will be inserted by the editor)

\title{
Continuum effects: Structure and reactions of ${ }^{6} \mathrm{He}$
}

\author{
M. Rodríguez-Gallardo ${ }^{1,2,3}$ a , J. M. Arias ${ }^{2}$, J. Gómez-Camacho², R. C. Johnson ${ }^{3}$, A. M. Moro², I. J. Thompson ${ }^{3}$, \\ and J. A. Tostevin ${ }^{3}$ \\ 1 Centro de Física Nuclear, Universidade de Lisboa, Av. Prof. Gama Pinto 2, 1649-003 Lisboa, Portugal \\ 2 Departamento de Física Atómica, Molecular y Nuclear, Universidad de Sevilla, Apartado 1065, 41080 Sevilla, Spain \\ 3 Department of Physics, University of Surrey, Guildford GU2 7XH, United Kingdom
}

Received: date / Revised version: date

\begin{abstract}
A description of three-body weakly bound systems using the Transformed Harmonic Oscillator (THO) method is addressed. First, relevant structure observables are presented for the Borromean nucleus ${ }^{6} \mathrm{He}$. Then, the THO method is applied to the study of ${ }^{6} \mathrm{He}$ scattering within the Continuum-Discretized Coupled Channels (CDCC) framework.
\end{abstract}

PACS. 21.45. $+\mathrm{v}-21.10 .-\mathrm{k}-27.20 .+\mathrm{n}-24.10 .-\mathrm{i}-24.10 . \mathrm{Eq}-25.60 .-\mathrm{t}-25.60 . \mathrm{Bx}$

\section{Introduction}

A particularly interesting example of weakly bound systems is that of Borromean nuclei, that is, three-body composite systems with no binary bound subsystems. For an appropriate description of the reactions induced by a Borromean nucleus, like ${ }^{6} \mathrm{He}$, the introduction of the continuum part of the spectrum is necessary. For this purpose it is required to discretize the three-body continuum. This work has been developed by the group of M. Kamimura [1, $2]$ using a discretization method based on Gaussian functions. Here, an alternative discretization method, based on Hyperspherical Harmonics and orthogonal polynomials, is presented.

\section{Three-body continuum discretization method: Application to ${ }^{6} \mathrm{He}$}

This formalism is a generalization of the method presented in Ref. [4] for a two-body system. For a three-body case the hyperspherical coordinates $\{\rho, \alpha, \widehat{x}, \widehat{y}\}$ will be used. They are obtained from the Jacobi coordinates $\{\boldsymbol{x}, \boldsymbol{y}\}$ (see, for instance, Ref. [8]). The Jacobi coordinates are presented in Fig. 1.

First, the wave functions of the system are expanded in Hyperspherical Harmonics (HH) as

$$
\Psi_{\beta j \mu}(\rho, \Omega)=R_{\beta j}(\rho) \sum_{m \sigma}\left\langle l m S_{x} \sigma \mid j \mu\right\rangle \Upsilon_{K l m}^{l_{x} l_{y}}(\Omega) \chi_{S_{x}}^{\sigma}
$$

where $R_{\beta j}(\rho)$ is the hyperradial wave function, $\chi_{S_{x}}^{\sigma}$ the spin wave function of the two particles related by the co-

\footnotetext{
a Present address: Centro de Física Nuclear, Universidade de Lisboa
}

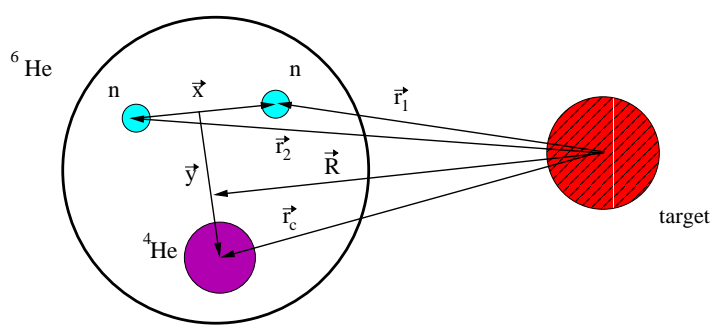

Fig. 1. Jacobi coordinates; ${ }^{6}$ He scattered by a target.

ordinate $\boldsymbol{x}$, and $\Upsilon_{K l m}^{l_{x} l_{y}}(\Omega)$ are the hyperspherical harmonics that depend on the angular variables $\Omega \equiv\{\alpha, \widehat{x}, \widehat{y}\}$. The set of quantum numbers $\beta \equiv\left\{K, l_{x}, l_{y}, l, S_{x}\right\}$ defines each channel. Here, $K$ is the hypermomentum, $l_{x}$ and $l_{y}$ are the orbital angular momenta associated with the Jacobi coordinates $\boldsymbol{x}$ and $\boldsymbol{y}, \boldsymbol{l}=\boldsymbol{l}_{x}+\boldsymbol{l}_{y}$ is the total orbital angular momentum, $S_{x}$ is the spin of the particles related by the coordinate $\boldsymbol{x}$ and $\boldsymbol{j}=\boldsymbol{l}+\boldsymbol{S}_{x}$ is the total angular momentum.

Then the Transformed Harmonic Oscillator method (THO) [3] is used to obtain $R_{\beta j}(\rho)$. The basic idea of the THO method [4-7] is to convert the bound groundstate wave function of the system into the ground-state wave function of the Harmonic Oscillator (HO), defining a Local Scale Transformation (LST). Considering that the ground-state wave function can be written as a linear combination of the basis functions given in Eq. (1), the equation that defines the LST for every channel $\beta$ is

$$
\int_{0}^{\rho} d \rho^{\prime} \rho^{\prime 5}\left|R_{B \beta}\left(\rho^{\prime}\right)\right|^{2}=\int_{0}^{s} d s^{\prime} s^{\prime 5}\left|R_{0 K}^{H O}\left(s^{\prime}\right)\right|^{2},
$$

where $R_{0 K}^{H O}(s)$ is the hyperradial wave function of the $\mathrm{HO}$. Finally, the THO basis is constructed for each channel 

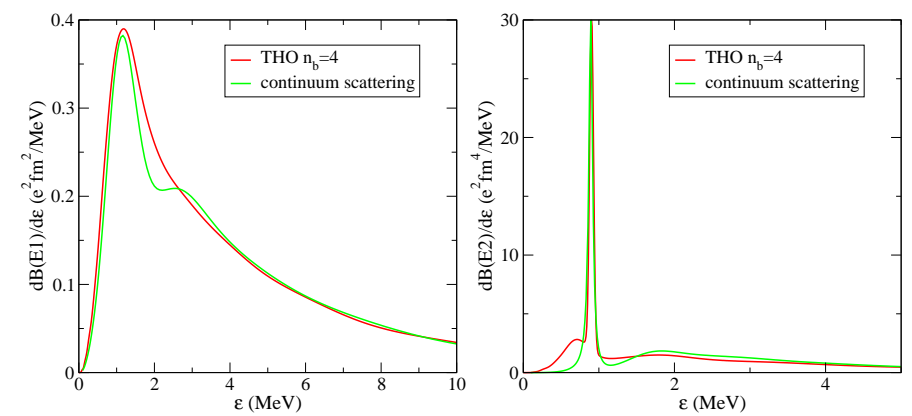

Fig. 2. (left) $B(E 1)$ distribution for $n_{b}=4$. (right) $B(E 2)$ distribution for $n_{b}=4$.

applying the LST, $s_{\beta}(\rho)$, to the HO basis

$$
R_{i \beta}^{T H O}(\rho)=R_{B \beta}(\rho) L_{i}^{K+2}\left(s_{\beta}(\rho)^{2}\right)
$$

where $L_{i}^{\lambda}(t)$ are Laguerre polynomials. In this way, a complete and orthonormal basis is obtained. Here the quantum number $i$ denotes the hyperradial excitations. Note that as $i$ increases, the functions $R_{i \beta}^{T H O}(\rho)$ become more oscillatory and explore larger distances.

Next we apply the method to the Borromean nucleus ${ }^{6} \mathrm{He}$. In that case the Hamiltonian $(\mathrm{H})$ is detailed in [8] and includes two-body interactions plus an effective threebody potential and a treatment of Pauli principle using a pseudopotential. $\mathrm{H}$ is diagonalized in a finite THO basis with $N$ states (including $n_{b}$ hyperradial excitations in every channel so $N=\left(n_{b}+1\right) \times n$ chan, where nchan is the number of channels for each state).

For $j=0^{+}$we get almost exactly the ground-state of the system. For $j=2^{+}$and $n_{b}<5$, we obtain a very stable state around the experimental energy of the $2^{+}$resonance for ${ }^{6} \mathrm{He}$.

In Fig. 2 we present the $B(E 1)$ and $B(E 2)$ distributions for $n_{b}=4$ as a function of the excitation energy on the continuum. For comparison, the distribution calculated with the continuum scattering wave functions, taken from Ref. [9], is also shown. For both cases, $B(E 1)$ and $B(E 2)$, the two distributions are in good agreement with the same total strength. For the $B(E 2)$ distribution the most relevant feature is the presence of a narrow low-lying resonance.

\section{Reactions induced by ${ }^{6} \mathrm{He}$}

In Fig. 1 we show a scheme of the scattering of ${ }^{6} \mathrm{He}$ by a target, where $\boldsymbol{R}$ is the position of the center of mass of ${ }^{6} \mathrm{He}$ from the target. In order to perform CDCC [10] calculations, we take as the projectile basis, the eigenstates obtained from the diagonalization of $\mathrm{H}$ in a THO basis with a given numbers of states $(N)$. Then it is necessary to calculate the coupling potentials $V_{L n j, L^{\prime} n^{\prime} j^{\prime}}^{J}(R)=$ $\left\langle L n j J M\left|V_{1 t}\left(\boldsymbol{r}_{1}\right)+V_{2 t}\left(\boldsymbol{r}_{2}\right)+V_{c t}\left(\boldsymbol{r}_{c}\right)\right| L^{\prime} n^{\prime} j^{\prime} J M\right\rangle$. These potentials are read externally in the program FRESCO [11], that solves the system of coupled equations as in the conventional CDCC method. As a preliminary result we show

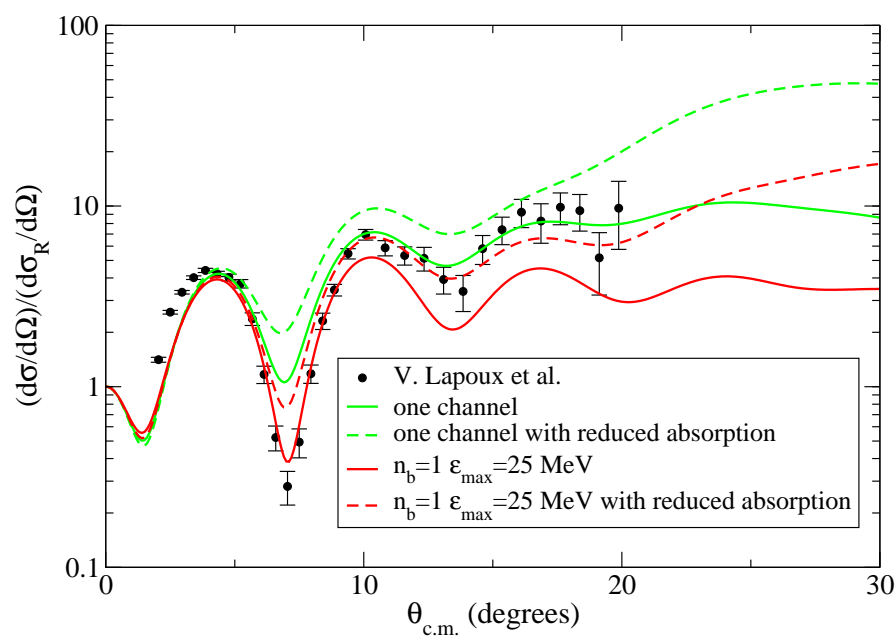

Fig. 3. Differential elastic cross section relative to Rutherford cross section for the reaction ${ }^{6} \mathrm{He}+{ }^{12} \mathrm{C}$ at $229.8 \mathrm{MeV}$.

in Fig. 3 the differential elastic cross section relative to Rutherford cross section for the reaction ${ }^{6} \mathrm{He}+{ }^{12} \mathrm{C}$ at 229.8 $\mathrm{MeV}$. We can see how the calculation with a very small THO basis reproduces quite well the experimental data of V. Lapoux et al. [12]. In order to reproduce satisfactory the normalization of the data for angles beyond $10^{\circ}$ we had to reduce the imaginary part of the coupling potentials by around $40 \%$. A similar reduction was also required in the work [1].

The purpose, in the next future, is the application of this procedure to analyze scattering cross sections of halo nuclei.

\section{Acknowledgments}

This work was supported in part by the DGICYT under projects FIS2005-01105 and FPA 2005-04460. M.R.G. acknowledges a FCT grant POCTI/ISFL/2/275 and FCT under the grant POCTI/FIS/43421/2001.

\section{References}

1. T. Matsumoto et al., Phys. Rev. C 70 (2004) 061601.

2. T. Matsumoto et al., Phys. Rev. C 73 (2006) 051602(R).

3. I. Zh. Petkov and M. V. Stoitsov, Compt. Rend. Bulg. Acad. Sci. 34 (1981) 1651.

4. F. Pérez-Bernal et al., Phys. Rev. A 63 (2001) 052111.

5. I. Martel et al., Phys. Rev. A 65 (2002) 052708.

6. M. Rodríguez-Gallardo et al., Phys. Rev. C 69 (2004) 034308.

7. M. Rodríguez-Gallardo et al., Phys. Rev. C 72 (2005) 024007.

8. I. J. Thompson et al., Comput. Phys. Commun.161 (2004) 87.

9. I. J. Thompson et al., Phys. Rev. C 61 (2000) 24318.

10. N. Austern et al., Phys. Rep. 154 (1987) 125.

11. I. J. Thompson, Comput. Phys. Rep. 7 (1988) 167.

12. V. Lapoux et al., Phys. Rev. C 66 (2002) 034608. 